Estudios Constitucionales, Año 12, No 2, 2014, pp. 13-28.

ISSN 07180195

Centro de Estudios Constitucionales de Chile Universidad de Talca

"Los deberes de las personas y la realización de los derechos fundamentales"

Adriano Sant'Ana Pedra

\title{
LOS DEBERES DE LAS PERSONAS Y LA REALIZACIÓN DE LOS DERECHOS FUNDAMENTALES*
}

\author{
THE DUTIES OF THE PERSONS AND \\ THE EFFECTIVENESS OF THE FUNDAMENTAL RIGHTS
}

\author{
Adriano SAnT'Ana Pedra** \\ Faculdade de Direito de Vitória \\ adrianopedra@fdv.br
}

RESUMEN: El estudio evalúa los comportamientos que puedan exigirse de las personas con el fin de garantizar los derechos fundamentales. Así, la investigación se realiza desde el papel de los deberes fundamentales de la persona humana en la realización de los derechos fundamentales. El estudio se ocupa del deber fundamental no como una práctica egoista e individualista, sino más bien desde una perspectiva en la que se respete e incluya al otro. Desde la perspectiva de la alteridad, el comportamiento ético requiere que se ponga al servicio de los demás, lo que se traduce en el respeto de los derechos fundamentales.

ABSTRACT: This study aims to analyze behaviors that may be required from people to ensure fundamental rights. Thus, the research is taken from the role of fundamental human duties in order to accomplish fundamental rights. The study takes into account an approach to the fundamental duty not as a selfish and individualistic provision, but rather from a perspective in which the other is respected and included. From the perspective of otherness, ethical behavior causes that someone protect the other, which results in the accomplish of fundamental rights.

PALABRAS CLAVE: Deber fundamental, Derecho fundamental, Solidaridad.

KEYWORDS: Fundamental duty, Fundamental right, Solidarity.

\section{Nota INTRODUCTORIA}

"Se habla de derechos humanos y está bien, y hay que seguir hablando, pero hablamos poco de los deberes humanos. ¿Deberes de qué? De solidaridad, sobre todo. De respeto humano, sobre todo. Estamos olvidando un poco que los derechos se

\footnotetext{
* Trabajo recibido el 24 de febrero de 2014 y aceptado el 1 de octubre de 2014.

** Doctor en Derecho Constitucional (Pontifícia Universidade Católica de São Paulo-PUC/SP). Máster en Derechos y Garantías Fundamentales (Faculdade de Direito de Vitória-FDV). Profesor de Faculdade de Direito de Vitória (FDV) en los cursos de graduación, especialización, maestría y doctorado. Procurador Federal de Advocacia-Geral da União (Brasil).
} 
compaginan con los deberes. Hacerse cargo de la necesidad de hablar de esto es a lo que yo me refiero con 'compromiso ético' y 'compromiso crítico".

José Saramago

Las constituciones normalmente prescriben derechos fundamentales en el texto. Sin embargo, durante mucho tiempo, los estudios se han ocupado principalmente de los derechos fundamentales y ha habido algún abandono de los asuntos relativos a los deberes fundamentales de la persona humana, y esto es debido especialmente a la influencia liberal, con el desprecio de la solidaridad, así como debido al temor de que los deberes sirviesen a los regímenes autoritarios, como una reacción a los horrores cometidos contra la humanidad. En Brasil, el momento constituyente que sucedió a la dictadura militar ha hecho que el texto de la Constitución de 1988 fuese abundante en materia de derechos, pero muy pobre en materia de deberes.

Sin embargo, a los deberes fundamentales se reserva un papel noble. En muchas situaciones, la mera acción del Estado no es suficiente para garantizar los derechos fundamentales de una persona, lo que sólo puede ocurrir con la prestación de un deber por parte de otra persona. Esto sucede, por ejemplo, con el deber de la colectividad de defender y preservar el medio ambiente ecológicamente equilibrado.

En este estudio se pretende analizar el papel de los deberes humanos en la tutela de los derechos fundamentales. A pesar de la mención hecha a los deberes humanos, es decir, a los deberes fundamentales de la persona humana, el desarrollo construido aquí también se aplica a los deberes fundamentales de la persona jurídica, en lo que sea aplicable. Así, se evalúa la legitimidad de la exigibilidad de los deberes impuestos a los particulares por la Constitución con el fin de garantizar los derechos fundamentales.

La investigación tiene en cuenta el deber fundamental no como una prestación egoísta e individualista, sino desde una perspectiva en la que se respeta y se incluye el otro. Desde la perspectiva de la solidaridad, el comportamiento ético hace que cada uno esté al servicio del otro, lo que se traduce en la tutela de los derechos fundamentales.

\section{LOS DERECHOS Y LOS DEBERES}

FUNDAMENTALES EN UNA SOCIEDAD SOLIDARIA

Todo grupo social tiene un rango de valores, lo cual es importante para la caracterización de las diversas sociedades en el espacio y en el tiempo. La preferencia por ciertos valores está relacionada con el reconocimiento de la superioridad de un 
valor sobre otro. Aunque, en el ámbito del individuo, cada uno establezca sus preferencias y constituya una jerarquía subjetiva, en el ámbito de la sociedad debe haber una jerarquía común a todos sus miembros para que el Derecho consiga proteger a cada valor de acuerdo a su posición en esta escala ${ }^{1}$ Es necesario, por tanto, que los valores predominantes sean protegidos y, consecuentemente, sea garantizada la pacificación de los intereses de los miembros de la sociedad. Hacer esta protección es tarea del Derecho, que debe resolver los conflictos de interés y poner freno a la práctica de actos contrarios a los valores elegidos por esa sociedad. Por eso Miguel Reale dice que, "el Derecho existe porque hay la posibilidad de que sean violados los valores que la sociedad reconoce como esenciales para la convivencia" 2 .

La Constitución de 1988 tuvo un especial cuidado en garantizar los derechos fundamentales. Y no podría ser de otra manera, porque los derechos fundamentales son prerrogativas de las personas para garantizar una vida digna ${ }^{3}$. Además, desde el punto de vista formal, los derechos fundamentales son las matrices de todos los demás, dándoles fundamento, y sin ellos no se puede ejercer muchos otros. Los derechos fundamentales deben ser garantizados por la Constitución, que da origen y validez a todas las otras leyes que crean o garantizan los otros derechos ${ }^{4}$.

Las constituciones contemporáneas normalmente colocan los derechos y las garantías fundamentales en sus primeros títulos, mostrando que el Estado debe cumplir lo que está en él establecido. La Constitución brasileña de 1988 divide el Título II -Derechos y Garantías Fundamentales- en cuatro capítulos, a saber: derechos y deberes individuales y (artículo $5^{\circ}$ ), derechos sociales (artículos $6^{\circ}$ a 11), derechos de nacionalidad (artículos 12 y 13), derechos políticos (artículos 14 a 16) y los derechos relacionados con la existencia, organización y participación en los partidos políticos (artículo 17). Sin embargo, aún se pueden encontrar los derechos fundamentales en otras partes de la Constitución brasileña. Esta situación no se da sólo en el contexto brasileño, sino también en otros países.

Se puede verificar que la lista de los derechos fundamentales es más amplia en las constituciones de los países de democracia reciente que en las constituciones de los países de democracia consolidada, lo que ocurre, "por la necesidad de la protección del Estado a los bienes esenciales para la supervivencia de las poblaciones

\footnotetext{
1 Pedra (2012), p. 139.

2 Reale (1999), p. 189.

3 Pedra (2012), p. 115.

4 Salgado (1996), pp. 16-17.
} 
necesitadas" 5 . Sin embargo, como señala Norberto Bobbio, no siempre se puede realizar lo que es deseable y digno de ser perseguido, ya que "son frecuentemente necesarias condiciones objetivas que no dependen de la buena voluntad de los que la proclaman, o de las buenas disposiciones de los que tienen los medios para protegerlos" 6 .

Asimismo, no se puede olvidar que la satisfacción de las necesidades esenciales de las personas depende de acciones de otras personas (físicas o jurídicas), además de la actuación del Estado mismo. El derecho a la educación de un niño, por ejemplo, sólo puede estar plenamente asegurado con el cumplimiento del deber de los padres o tutores para con la educación de él (artículo 19, 10, de la Constitución de Chile), y eso ocurre con actuaciones como la inscripción en la escuela, el control de frecuencia y rendimiento escolar, entre otros. No es suficiente que el Estado provea escuelas, profesores, libros, cuadernos, transporte y comida, por ejemplo; es necesario que la familia participe de manera efectiva en la educación del niño.

Lo mismo ocurre con el derecho a la salud y las medidas contra el dengue o la fiebre amarilla, que dependen de la participación de todos para que no se formen criaderos de mosquitos que pueden transmitir las enfermedades. La acción del Estado únicamente no es suficiente para acabar con las enfermedades.

Por lo tanto, los deberes fundamentales tienen un papel importante en la protección y promoción de los derechos fundamentales; algunos de estos dependen directamente de aquellos (por ejemplo, el deber de educar a sus hijos), y otros dependen indirectamente (por ejemplo, el deber de pagar impuestos).

Quien tiene derechos también debe tener deberes. Esto se justifica con referencia a la reciprocidad: mi vecino respeta mi privacidad y quiere que yo respete la suya. Pero también se justifica con referencia a la solidaridad: hay que poner a la disposición de los grupos más débiles los recursos que permitan el ejercicio de los derechos fundamentales de manera satisfactoria, para fortalecer la cohesión social. Es un pensamiento que se opone al liberalismo-individualismo en su forma absoluta ${ }^{7}$.

De ahí la razón por la cual las personas deben ser solidarias. Una solidaridad que surge del ordenamiento jurídico y que no depende del altruismo de cada uno. La Constitución de Brasil pone como un objetivo fundamental de la República la construcción de "una sociedad libre, justa y solidaria" (artículo 3º, I). Las personas deben ser solidarias, y no solitarias, porque, además de la acción del Estado, son

5 TORres (2001), p. 286.

6 Воввіо (1992), pp. 44-45.

7 Dimoulis (2011), p. 339. 
necesarias conductas positivas y negativas de las personas para la protección de los derechos fundamentales.

La solidaridad es la manera de actuar que impele a la voluntad individual y colectiva para buscar conscientemente la satisfacción de las necesidades básicas del "otro". Dado que la solidaridad es un comportamiento consciente, es una cualidad que sólo pertenece a los seres humanos. Es necesario, por lo tanto, tener capacidad para el cumplimiento de un compromiso en relación con el "otro".

La solidaridad es un auténtico respeto por la especie humana y también su entorno natural y social. El "otro" no es alguien fuera de su vida. Es decir, las personas no sólo deben comportarse de manera tal que sus actuaciones no dañen al "otro", sino también la ética de los derechos humanos radica en la práctica de la solidaridad.

Así, la libertad debe ceder ante la solidaridad. Este ceder no significa que se esté vulnerando la libertad de una persona, sino lo contrario, la solidaridad garantizará la libertad de todos. Pero es preciso que las personas renuncien al egoísmo.

Sin embargo, la solidaridad no es caridad. La caridad nos obliga a ayudar a los otros por amor, sin que el otro tenga el derecho a una limosna o ayuda. En cambio, la solidaridad (normativa) no es un regalo, sino que es un derecho del "otro".

\section{LA FUNDAMENTALIDAD DE LOS DEBERES HUMANOS}

Y LA EXIGENCIA DE ESFUERZOS PERSONALES PARA LA PROTECCIÓN Y

LA PROMOCIÓN DE LOS DERECHOS FUNDAMENTALES

Debido a la meta de construir una sociedad libre, justa y solidaria, las personas de esta sociedad deben tener conductas compatibles con la realización de estos valores. Por eso la prescripción, por la Constitución, de deberes fundamentales compatibles con la promoción de estos ideales.

Así, por un lado, se requiere una prescripción constitucional (fundamentalidad formal) sobre los deberes fundamentales, ya que éstos deben ser establecidos por normas con rango constitucional. Según Gregorio Peces-Barba Martínez, "el deber jurídico tiene que estar reconocido por una norma perteneciente al ordenamiento" 8 . Esta es una razón lógica, de inserción en la Constitución, creada por el poder constituyente (originario o derivado) y que surge de la soberanía popular.

Vale la pena señalar que la Constitución puede establecer deberes fundamentales tanto implícita como explícitamente, y también, así como los derechos, otros

8 Peces-Barba Martínez (1987), p. 335. 
derivados del régimen y de los principios adoptados por la Constitución, y de los tratados internacionales ${ }^{9}$ en que la República Federativa de Brasil sea parte, en virtud del artículo $5^{\circ}, \$ 2^{\circ}$, de la Constitución brasileña, lo que nos lleva a la idea de bloque de constitucionalidad ${ }^{10}$.

Por otro lado, la fundamentalidad material tiene en cuenta la relevancia del deber hacia la satisfacción de las necesidades básicas esenciales de una persona -de sí mismo, de otras personas o de la comunidad- con el objetivo de defensa y promoción de los derechos fundamentales.

Por lo tanto, se puede decir que los deberes fundamentales se vinculan directamente "a la necesidad de los hombres - seres gregarios por naturaleza- de vivir en la comunidad, lo que exige la contribución de todos para que los objetivos comunes sean alcanzados" 11 .

De hecho, los autores que se concentran en el tema de los deberes fundamentales señalan que es necesario entenderlos no como un contrapunto o una mitigación de los derechos, sino como un promotor de éstos.

Según José Casalta Nabais:

"Los deberes fundamentales constituyen una categoría jurídico-constitucional propia colocada al lado y correlativa de la categoría de los derechos fundamentales, una categoría que, como correctivo de la libertad, refleja la movilización del hombre y del ciudadano a la consecución del bien común"12.

A pesar de ser una categoría autónoma, los deberes fundamentales están correlacionados con los derechos fundamentales, ya que éstos son no solo limitados sino también garantizados por aquellos ${ }^{13}$. De hecho, se justifica el vínculo entre derechos y deberes fundamentales, porque el derecho de un individuo lleva al surgimiento de al menos un deber para los demás ${ }^{14}$, que puede ser el deber de no impedir el ejercicio del derecho o incluso el de su promoción.

En este momento, es importante traer el concepto de deber fundamental adoptado en este estudio:

9 Véase, por ejemplo, el deber de instrucción establecido por el artículo XXXI de la Declaración Americana de los Derechos y Deberes del Hombre, aprobada en la Novena Conferencia Internacional Americana, en Bogotá (1948): “Toda persona tiene el deber de adquirir a lo menos la instrucción primaria”.

10 Vieira y Pedra (2013), p. 9.

11 Schwan y Pedra (2011), p. 178.

12 Nabais (2009), p. 64.

13 Duque y Pedra (2012), p. 18.

14 Gonçalves y Pedra (2011), p. 209. 
Deber fundamental es una categoría jurídico-constitucional, fundada en la solidaridad, que impone conductas proporcionales a los que están sometidos a un orden democrático, susceptible de sanción o no, con la finalidad de promoción de derechos fundamentales ${ }^{15}$.

Cabe destacar que el deber impuesto a alguien no puede ser un esfuerzo excesivo para él. Aunque en razón de la idea de solidaridad, no se puede imponer al sujeto del deber un sacrificio extraordinario -o desproporcional- para salvaguardar cierto derecho. Sólo se puede exigir de ese sujeto un "sacrificio trivial"16.

La carga impuesta sobre el sujeto del deber no puede ser exorbitante, lo que es una prohibición que se debe evaluar en cada caso concreto, jamás en abstracto, ponderándose lo que se intenta alcanzar con esta obligación.

En general, se puede requerir a alguien que salve a un niño que se está ahogando frente a él en una piscina de poca profundidad, pero lo mismo no puede requerir si el ahogamiento ocurre en un mar tormentoso, porque, en este caso, el sujeto del deber pondría su propia vida en peligro. El delito de omisión de socorro (artículo 135 del Código Penal brasileño) debe tener en cuenta la situación concreta. Del mismo modo, cuando hay una situación de pobreza extrema y ausencia de condiciones para cumplir con el deber fundamental de garantizar la educación de sus hijos (artículos 205, 227 y 229 de la Constitución brasileña), no se puede condenar penalmente a los padres que no proveen la educación primaria del niño en edad escolar, lo que podría caracterizar, en una situación diferente, crimen de abandono intelectual (artículo 246 del Código Penal brasileño). En el ámbito fiscal, el principio de la capacidad de pago es un corolario que surge de la prohibición de carga excesiva -o exorbitante- del sujeto del deber.

Por lo tanto, la trivialidad del esfuerzo dependerá no sólo del sujeto del deber sino también del derecho fundamental correlacionado que se intenta proteger, lo que hace posible hablar de ponderación subjetiva y de ponderación objetiva, respectivamente. Una carga tributaria puede ser excesiva o no, dependiendo de la capacidad del sujeto contribuyente (ponderación subjetiva) o dependiendo de existir una situación de guerra o de paz (ponderación objetiva), por ejemplo.

Es necesario que haya equidad en la carga asumida por cada persona. Especialmente en aquellas situaciones en las que la carga se concentra específicamente en

15 Concepto construido colectivamente por los miembros del Grupo de Investigación "Estado, Democracia Constitucional y Derechos Fundamentales" (2013), coordinado por los profesores Adriano Sant'Ana Pedra y Daury Cesar Fabriz, de Faculdade de Direito de Vitória-FDV (Estudios de Postgrado-Maestría y Doctoradoen Derechos y Garantías Fundamentales).

16 GaRzón (1986), p. 17. 
algunos pocos individuos, y la sociedad es beneficiaria, el ordenamiento jurídico debe prescribir, en la medida de lo posible, la redistribución de esa carga con los otros miembros de la sociedad ${ }^{17}$.

Esto es lo que sucede, por ejemplo, cuando hay una reducción de la carga fiscal en caso de inventariado como de interés histórico y cultural (deber fundamental de defender y preservar el patrimonio histórico y cultural, en virtud del artículo 216, $\$ 1^{\circ}$, de la Constitución brasileña), o igualmente en caso de mantenimiento de reservas forestales (deber fundamental de defender y preservar el medio ambiente ecológicamente equilibrado, establecido en el artículo 225 de la Constitución brasileña). Dichas compensaciones deben producirse para que la sociedad también soporte dichos gravámenes con el financiamiento de la protección de bienes constitucionalmente protegidos.

\section{Deberes COMO NORMAS DE BAJA DENSIDAD NORMATIVA \\ Y LA CUESTIÓN DE LA (IN)NECESIDAD DE INTEGRACIÓN LEGISLATIVA}

Las normas constitucionales que establecen derechos fundamentales son, en general, normas con baja densidad normativa, lo que, dicho sea de paso, es propio de las normas constitucionales.

Las normas que establecen deberes autónomos de los particulares suelen tener baja densidad normativa. En nuestro ejemplo, la Constitución no dice lo que la "familia" debe hacer para promover la educación de sus miembros, cuáles integrantes de la familia deben asumir esta obligación y cómo, si la familia debe limitarse a cuidar de la formación de sus miembros, si es suficiente inscribir a los jóvenes en las instituciones educativas o si la misma familia debe proporcionarles conocimientos, etc. ${ }^{18}$.

De hecho, debido a su naturaleza y función, los textos constituciones son más abiertos que aquellos que sirven al ordenamiento infraconstitucional. El lenguaje utilizado en el texto constitucional es dotado de un elevado grado de abstracción

17 Para que los medios sean proporcionados al individuo para que cumpla con su deber, en algunos casos la carga del sujeto del deber no es compartida con la sociedad, sino con ciertas personas, como, por ejemplo, con su empleador y con la parte en un proceso. Véase, por ejemplo, el artículo 419 del Código de Proceso Civil brasileño: "Artículo 419.- El testigo puede solicitar al juez el pago de los gastos realizados para asistir a la audiencia, y la parte debe pagar prontamente cuando arbitrada, o depositar en la secretaría del juicio dentro de los 3 (tres) días. Parágrafo único. Se considera servicio público el testimonio prestado en juicio. El testigo, cuando se somete a las leyes laborales, no sufre, para asistir a la audiencia, pérdida de salario ni descuento en el tiempo de servicio".

18 Dimoulis y Martins (2011), p. 330. Ver también: Dimoulis y Martins (2012), p. 63. 
semántica que "permite al operador normativo actuar con más alto grado de libertad en la determinación de la extensión y de la profundidad de los institutos insertos en el cuerpo de la Norma Fundamental"19. Debido a su estructura y función, "la norma constitucional casi siempre aparece más indefinida y fragmentaria que las otras normas de los sistemas jurídicos dogmáticos modernos" 20 .

La naturaleza del lenguaje constitucional, adecuado para acarrear normas principiológicas y esquemáticas, hace que éstas presenten mayor apertura, mayor grado de abstracción y, por tanto, menor densidad jurídica. Conceptos tales como igualdad, moralidad, función social de la propiedad, justicia social, bien común, dignidad humana, entre otros, dan al intérprete un espacio significativo de discreción. El problema de esa libertad de conformación en la interpretación judicial es más agudo en los países de Constitución sintética, donde la plasticidad de ciertas cláusulas genéricas admite variaciones entre extremos. Sin embargo, incluso en los Estados que adoptan una Carta analítica -como en el caso de Brasil- la cuestión se plantea con frecuencia ${ }^{21}$.

Además, al dejar, conscientemente, de regular ciertas tareas, al optar por una técnica normativa de normas abiertas, la Constitución forma un instrumento democrático que posibilita confrontaciones políticas ${ }^{22}$.

Por otra parte, la apertura del sistema constitucional expresa el carácter incompleto y la precariedad del conocimiento científico ${ }^{23}$. El jurista, como cualquier científico, debe estar dispuesto a poner en cuestión el sistema para ampliarlo o modificarlo en base a una mejor consideración. Con esto en mente, la textura abierta del lenguaje es una ventaja, ya que toma en cuenta tanto la necesidad de certeza cuanto la necesidad de dejar algunas cuestiones abiertas para que sean evaluadas en el momento adecuado.

Dimitri Dimoulis y Leonardo Martins traen ejemplos de la Constitución española, que establece que "los españoles tienen el derecho y el deber de defender a España" (artículo 30, I), y la Constitución italiana, que establece que: "la defensa de la Patria es deber sagrado del ciudadano" (artículo 52, I), y se preguntan: “¿Qué significa 'defender' un país? ¿Defender de quién? ¿Y cómo? ¿Militarmente?

\footnotetext{
19 Santos (2008), p. 195.

20 Adeodato (2006), p. 218.

21 Barroso (2002), pp. 107-108.

22 Pedra (2012), p. 35.

23 Canaris (1996), p. 106.
} 
¿Económicamente? ¿Políticamente? ¿Con la promoción del arte, de la literatura, o-¿por qué no?- de la cocina nacional?’24.

Por eso hay necesidad (relativa) de integración legislativa.

Aunque haya textos normativos redactados de forma clara y precisa, incluso éstos deben ser interpretados para aclarar mejor el contenido, porque la norma jurídica no es aplicable como quiera el positivismo con base en la claridad del texto ${ }^{25}$. Los textos normativos requieren interpretación no sólo debido a que no son obvios o unívocos, es decir, carentes de la claridad, sino también debido a que deben ser aplicados a situaciones $\operatorname{concretas}^{26}$. En ese sentido ha entendido Karl Larenz:

Sería un error aceptar que los textos jurídicos requieren interpretación solamente cuando sean particularmente "obscuros", "poco claros" o "contradictorios". En principio, todos los textos jurídicos son susceptibles y requieren interpretación. Esta su necesidad de interpretación no es un "defecto" que se podría remediar en definitiva a través de una redacción lo más precisa posible ${ }^{27}$.

El carácter indispensable de la interpretación se debe a que el sistema jurídico deja varias posibilidades abiertas y no contiene todavía una decisión de cuál de los intereses en conflicto es el valor más importante.

La regulación genérica de esos deberes por la Constitución desempeña un doble papel. Por una parte, guía al legislador ordinario para que, en el ejercicio de sus funciones, reglamente los deberes, ya que tienen baja densidad normativa. Por otro lado, la regulación constitucional es el fundamento para el examen de la constitucionalidad de esa legislación. Tenemos así una estructura bifásica del deber fundamental ${ }^{28}$.

Hay que añadir que la supuesta claridad no es una propiedad del texto, sino el resultado de la interpretación. Es decir, para afirmar que un enunciado normativo es claro, es necesario interpretarlo.

Se observó que los deberes fundamentales tienen por objeto garantizar derechos fundamentales. Por lo tanto, la inercia del legislador para establecer los comportamientos obligatorios al sujeto del deber perjudica a los derechos fundamentales que deberían ser protegidos por tales conductas.

24 Dimoulis (2011), pp. 342-343.

25 SiLVA (2000), p. 417.

26 Grau (2006), p. 29.

27 LARENZ (1997), p. 283-284.

28 Dimoulis y Martins (2011), p. 335. 
Hay, por ejemplo, una omisión inconstitucional del Poder Legislativo federal brasileño, que no instituye el impuesto sobre grandes fortunas previsto en el artículo 153, VII, de la Constitución de Brasil, lo que impide la implementación de los muchos beneficios para la población -ya que los recursos públicos son escasos y los derechos tienen $\operatorname{costos}^{29}$. La no creación de este impuesto no es un alivio para el contribuyente, sino una gran injusticia, porque los contribuyentes que no tienen "grandes fortunas" se quedan más cargados de lo que deberían. El legislador solamente podría dejar de instituir el mencionado tributo si no fuese necesario; pero eso no es verdad. Incluso en la situación hipotética y poco probable en que los ingresos públicos fuesen suficientes para satisfacer todas las demandas de la sociedad, sin embargo, el impuesto sobre las grandes fortunas debería ser instituido para aliviar a los contribuyentes menos afortunados que pagan los impuestos sobre el consumo, sobre la renta de trabajo, etc.

Podemos señalar muchas otras omisiones inconstitucionales del legislador para regular conductas al sujeto del deber, e incluso para establecer sanciones en caso de incumplimiento, que servirían para proteger y promover los derechos fundamentales. Sin embargo, a pesar de las voces disonantes ${ }^{30}$, el neoconstitucionalismo indica una opinión de que incluso las normas constitucionales que dicen respecto a deberes fundamentales deben aplicarse directamente, sin la (necesaria) intermediación del legislador ordinario, tanto como sea necesario y posible.

Es importante traer el ejemplo de la construcción judicial hecha por el Supremo Tribunal Federal brasileño (ADPF No 132/RJ y la ADI No 4.277/DF), que reconoció que el régimen jurídico de las uniones estables se aplica también a las uniones homosexuales, haciéndose una nueva lectura del artículo $226, \S 3^{\circ}$, de la Constitución. Además del reconocimiento de los derechos inherentes a la unión estable, hubo también la creación de deberes fundamentales -como, por ejemplo, los deberes conyugales (artículo $226, \$ 5^{\circ}$, de la Constitución) de ayuda mutua- para las personas en unión estable homosexual, lo que no existía hasta la decisión del Tribunal Supremo en mayo de 2011.

Sin embargo, es necesaria la mediación legislativa para imponerse penas por incumplimiento de algún deber ${ }^{31}$, porque normalmente tales sanciones no están

29 Cf. Holmes (2011).

30 Cf. Chulvi (2001), p. 49. Véase también: Rubio (2001), p. 21. Cf. también: Dimoulis y Martins (2012), p. 66.

31 SARLET (2007), p. 244. 
previstas en la Constitución, lo que no puede tener efectos retroactivos ${ }^{32}$. La pena es importante porque es un elemento coercitivo, pero no es esencial para la eficacia de un deber fundamental. Pero, incluso sin sanciones, es normal que haya consecuencias jurídicas como resultado del incumplimiento de un deber fundamental, dada su normatividad.

\section{CONSIDERACIONES FINALES}

Para muchas personas, la palabra "deber" todavía se refiere a la idea de limitación de derechos, castración de libertades y autoritarismo estatal ${ }^{33}$. De hecho, los deberes fundamentales restringen las libertades de la persona a quién se impone el deber. Sin embargo, el estudio de los deberes fundamentales ha servido para mostrar la otra cara de la moneda: los deberes se prestan para tutelar los derechos fundamentales ${ }^{34}$. Además, la consolidación de los estudios sobre los deberes fundamentales destaca la necesidad de sopesar el sacrificio de cada persona para que no haya exorbitancias en las prestaciones positivas o negativas requeridas del sujeto del deber ${ }^{35}$.

El ordenamiento jurídico debe prever prestaciones alternativas para aquellas personas que no pueden cumplir con el comportamiento requerido originalmente, cuando hay una carga excesiva a la persona, como ocurre por ejemplo en los casos de objeción de conciencia en razón de creencias religiosas y de convicciones filosóficas o políticas.

Las personas cumplen con el deber y conforman su comportamiento, cuando se dan cuenta que las prestaciones que se requieren son legítimas.

32 Bernardo y Pedra (2011), p. 203.

33 Tavares y Pedra (2013), pp. 203-223. Véase también: Tavares y Pedra (2012), p. 170.

34 Cf. ponencia "Los deberes fundamentales y la tutela efectiva de los derechos", de este autor, presentada en el "XI Congreso Iberoamericano de Derecho Constitucional" (San Miguel de Tucumán, Argentina, 2013), organizado por el Instituto Iberoamericano de Derecho Constitucional, por la Asociación Argentina de Derecho Constitucional y por la Facultad de Derecho y Ciencias Sociales de la Universidad Nacional de Tucumán.

35 Véase, por ejemplo, los artículos 347 y 406 del Código de Proceso Civil brasileño: “Artículo 347.- La parte no está obligada a prestar declaración de los hechos: I.- criminales o torpes, que se asignan; II.- de que, por estado o profesión, debe guardar secreto. Parágrafo único. Esta disposición no se aplica a las acciones de filiación, de separación y de nulidad de matrimonio". "Artículo 406.- El testigo no está obligado a prestar declaración de fatos: I.- que le causen perjuicio grave, así como a su cónyuge y a sus parientes por consanguinidad o afinidad, hasta el segundo grado inclusive; II.- de que, por estado o profesión, debe guardar secreto". 
Sin embargo, en Brasil, verifícase la ausencia de un sentimiento constitucional debido a un escenario legislativo alejado de la realidad social y de raíces heredadas del proceso de colonización, con la imposición de normas del ordenamiento jurídico por parte del gobierno, sin ningún tipo de interacción o manifestación social, lo que genera un sentimiento de no pertenencia ${ }^{36}$.

Es recurrente el ejemplo de los impuestos. En "tierras tupiniquins", la resistencia al deber de contribuir con los gastos públicos se da por varios factores, entre ellos la falta de discusión pública y transparencia en la asignación de los montos recaudados, la elevada carga fiscal, la mala gestión de los recursos y la corrupción que azota al país ${ }^{37}$.

Los individuos en general no respetan la ley si no la reconocen como legítima. Y eso significa que la persona debe darse cuenta de que los cargos moderados que se imponen se distribuyen más o menos por igual ${ }^{38}$ y contribuyen a la realización de los derechos fundamentales de sí, de su familia o de la comunidad a la que pertenece.

\section{REFERENCIAS}

Adeodato, João Maurício. (2006). Ética e retórica: para uma teoria da dogmática jurídica. Segunda edição (São Paulo: Saraiva).

Barroso, Luís Roberto. (2002). Interpretação e aplicação da Constituição: fundamentos de uma dogmática constitucional transformadora. Quarta edição. 2. tir. (São Paulo, Saraiva).

Bernardo Segundo, Ronaldo Louzada; Pedra, Adriano Sant'Ana. (2011). Limites ao dever de tolerância. In: Bussinguer, Elda Coelho de Azevedo. Direitos fundamentais: pesquisas. (Curitiba, CRV).

Bobbio, Norberto. (1992). A era dos direitos. Trad. Carlos Nelson Coutinho. (Rio de Janeiro, Campus).

Canaris, Claus Wilhelm. (1996). Pensamento sistemático e conceito de sistema na ciência do direito. Segunda edição. Trad. Antonio Menezes Cordeiro. (Lisboa. Calouste Gulbenkian).

Chulvi, Cristina Pauner. (2001). El deber constitucional de contribuir al sostenimiento de los gastos públicos. (Madrid, Centro de Estudios Políticos y Constitucionales).

36 Santos; Fittipaldi y Binda (2013), p. 248.

37 Machado y Pedra (2012), pp. 2-3.

38 Holmes (2011), p. 197. 
Dimoulis, Dimitri; Martins, Leonardo. (2011). Deveres fundamentais. In: Leite, George Salomão; Sarlet, Ingo Wolfgang; Carbonell, Miguel. Direitos, deveres e garantias fundamentais. (Salvador, Juspodivm).

Paulo, Atlas).

(2012). Teoria geral dos direitos fundamentais. Quarta edição (São

Duque, Bruna Lyra; Pedra, Adriano Sant'Ana. (2012). Os deveres fundamentais e a solidariedade nas relações privadas. In: Bussinger, Elda Coelho de Azevedo. Direitos e deveres fundamentais. (Rio de Janeiro, Lumen Juris).

GARZÓN VALDÉs, Ernesto. (1986). Los deberes positivos generales y su fundamentación. Doxa. No 3, pp. 17-33.

GonÇAlves, Luísa Cortat Simonetti; Pedra, Adriano Sant'Ana. (2011). Deveres fundamentais: a ressocialização enquanto dever do próprio apenado. In: BusSINGUER, Elda Coelho de Azevedo. Direitos fundamentais: pesquisas. (Curitiba, CR).

Grau, Eros Roberto. (2006). Ensaio e discurso sobre a interpretaçãolaplicação do direito. Quarta edição. (São Paulo. Malheiros).

Holmes, Stephen; Sunstein, Cass R. (2011). El costo de los derechos: por qué la libertad depende de los impuestos. Trad. Stella Mastrangelo. (Buenos Aires, Siglo Veintiuno).

LARENZ, Karl. (1997). Metodologia da ciência do direito. Trad. José Lamego. Terceira edição, (Lisboa, Calouste Gulbenkian).

Machado, Álvaro Augusto Lauff; Pedra, Adriano Sant'Ana. (2012). A redução das alíquotas do IPI incidente na comercialização de veículos automotores: uma (in)justificável relativização do dever fundamental de contribuir com os gastos públicos. In: Bussinger, Elda Coelho de Azevedo. Direitos e deveres fundamentais. (Rio de Janeiro: Lumen Juris).

NaBAIs, José Casalta. (2009). O dever fundamental de pagar impostos: contributo para a compreensão constitucional do estado fiscal contemporâneo. (Coimbra, Almedina).

Peces-Barba Martínez, Gregório. Los deberes fundamentales. Doxa. No 4. 1987. pp. 329-341.

Pedra, Adriano Sant'Ana. (2012). A Constituição viva: poder constituinte permanente e cláusulas pétreas na democracia participativa. Segunda edição, (Rio de Janeiro; Lumen Juris).

(2012). Mutação constitucional: interpretação evolutiva da Constituição na democracia constitucional. (Rio de Janeiro, Lumen Juris). 
Reale, Miguel. (1999). Filosofia do direito. Terceira edição (São Paulo, Saraiva). Rubio llorente, Francisco. (2001). Los deberes constitucionales. Revista Española de Derecho Constitucional. A. 21. No 62, mai./ago. 2001.

SAlgado, Joaquim Carlos. (1996). Os direitos fundamentais. Revista Brasileira de Estudos Políticos. No 82, jan., 1996. (Belo Horizonte, UFMG).

Santos, André Filipe Pereira Reis dos; FitTipaldi, Paula Ferraço; Binda, Rosana Júlia. (2013). O problema da legitimidade social do direito e da justiça numa sociedade desigual: considerações sociológicas a partir da realidade brasileira. Revista de Direitos Fundamentais e Democracia. Vol. XIII. No 13, jan./jun., 2013, pp. 235-249.

SAnTos, Sergio Roberto Leal dos. (2008). Manual de teoria da Constituição. (São Paulo, RT).

SARLET, Ingo Wolfgang. (2007). A eficácia dos direitos fundamentais. (Porto Alegre, Livraria do Advogado).

SCHWAN, Felipe Teixeira; PEDRA, Adriano Sant'Ana. (2011). A democracia brasileira e o dever fundamental de votar. In: BUSSINGUER, Elda Coelho de Azevedo (org.). Direitos fundamentais: pesquisas. (Curitiba, CRV).

SiLva, Kelly Susane Alflen da (2000). Hermenêutica jurídica e concretização judicial. (Porto Alegre, Sergio Antonio Fabris).

Tavares, Henrique da Cunha; Pedra, Adriano Sant'Ana. (2013). As obrigaçóes tributárias acessórias e a proporcionalidade na sua instituição: uma análise a partir da teoria dos deveres fundamentais. Revista Tributária e de Finanças Públicas. a. 21. Vol. CIX, mar./abr. 2013, pp. 203-223.

(2012). Obrigações tributárias acessórias na perspectiva do dever fundamental de contribuir com os gastos públicos: uma reflexão acerca dos critérios para sua instituição. In: Allemand, Luiz Cláudio da Silva (coord.). Direito tributário: questôes atuais. (Brasília, OAB).

TORRES, Ricardo Lobo. (2001). A cidadania multidimensional na era dos direitos. In: TORres, Ricardo Lobo (Org.). Teoria dos direitos fundamentais. Segunda edição (Rio de Janeiro, Renovar).

Vieira, Pedro Gallo; Pedra, Adriano Sant'Ana. (2013). O rol de deveres fundamentais na Constituição como numerus apertus. Derecho y Cambio Social. A. X. Vol. XXXI, jan./mar. 2013, pp. 1-14. 